Gemeinwesen / Communities 


\title{
Im Schutz der Mauern. Versicherheitlichung und pragmatische Differenzierung in der spätmittelalterlichen Stadt
}

\author{
Stefanie Rüther
}

\section{Abstract}

The citizens and inhabitants of late-medieval towns could be distinguished not only in terms of their social and legal status but also by the different lengths of time that they spent there. The relative permeability of urban space, the social heterogeneity of the town population as well as the inhabitants' high mobility became apparent in those moments when town authorities perceived these features to pose a problem and sanctioned them accordingly in the wake of immanent threats, for instance, in the event of war and insurgency or famine and epidemics. Such a security situation characterised the so-called town wars of southern Germany, which were waged in the southwest of the realm at the end of the 14th century between the princes, lords and the towns. The measures that the town authorities adopted to ensure town security attest to the tension between their attempt to limit the number of people within the town walls to a trusted group and the need to have a large pool of people available to fight the war. Among the latter group were aristocratic soldiers and mercenaries, merchants, Jews in the role of moneylenders or clergy who acted as informants or mediators. What the town authorities truly feared was the dissolution of their communities, the drifting apart of people and goods as well as the intrusion by other powers and actors and the mixing of their town populations with these groups.

The population was differentiated on the basis of the familiar markers applied prior to the crisis, such as financial and social status, estate affiliation, religious affiliation and gender. Yet during war time these categories were re-evaluated with an eye to security in that distinctions were made according to the extent to which an individual was potentially useful or dangerous on grounds of their connections or abilities. These forms of boundary drawing were an expression of pragmatic differentiation as they were temporary and geared toward the specific situation created by the war or other crises.

\section{Einleitung}

Für das Selbstverständnis der spätmittelalterlichen Städte war das Ideal der Freiheit und Gleichheit ihrer Bürger konstitutiv. Sie hoben sich damit 
gegen ihre auf Abhängigkeit und Ungleichheit ausgerichtete adelige Umwelt ab, an die sie zugleich durch ein dauerhaftes Konkurrenzverhältnis gebunden blieben (vgl. Isenmann 2014; Rüther 2009b). Die städtischen Obrigkeiten präsentierten ihre Gemeinden als geschlossene und homogene Einheiten (vgl. Frenz 2000, Meier 1994), und die aktuelle Stadtgeschichtsforschung perpetuiert diese Sichtweise, indem sie häufig in analytischer Verkürzung von der Stadt als Akteur spricht. Materiellen Ausdruck findet diese Geschlossenheit in den Stadtmauern, die - zum Schutz gegen ungewollte Eindringlinge und gewaltsame Angriffe errichtet - das befriedete Innere gegen ein feindliches Äußeres abgrenzten (vgl. Baeriswyl 2018; Greifenberg 2015; Krause 2019; Tracy 2000). Doch kann diese Vorstellung als eine handlungsleitende Fiktion gelten, von der die Städte im Alltag weit entfernt waren. Denn die Menschen hinter den Stadtmauern unterschieden sich nicht nur durch ihren sozialen und rechtlichen Status, auch ihre Anwesenheit in der Stadt war von unterschiedlicher Dauer und ebenso verschieden motiviert wie legitimiert (vgl. Dilcher 1998).

Die relative Offenheit des städtischen Raums, die soziale Heterogenität seiner Bewohner*innen sowie deren hohe Mobilität werden in den Momenten fassbar, in denen sie angesichts einer drohenden Gefahr, etwa durch Krieg und Aufruhr, Hunger oder Seuchen, als Problem wahrgenommen und durch die städtischen Obrigkeiten sanktioniert wurden. Eine solche Sicherheitssituation stellen die sogenannten Süddeutschen Städtekriege dar, die am Ende des 14. Jahrhundert im Südwesten des Reiches zwischen Fürsten, Herren und Städten ausgetragen wurden (vgl. Rüther 2009; Schubert 2003; Schneider 1999; Holtz 1993). Bereits vor dem Beginn der kriegerischen Gewalt ergriffen die städtischen Magistrate eine Reihe von Maßnahmen, mit denen sie Sicherheit im Inneren der Städte angesichts der drohenden Kriegsgefahr zu gewährleisten suchten. Sie unterteilten Bürger*innen, Einwohner*innen und Gäste in verschiedene Gruppen, denen sie ein unterschiedliches $\mathrm{Ma}$ an ,sicher sein' zuerkannten. Die damit verbundenen Praktiken und Medien der Einteilung und Grenzziehungen stehen im Mittelpunkt des folgenden Beitrags. Sie betrafen nicht allein sozial prekäre Gruppen, wie Bettler*innen und Tagelöhner*innen, sondern konnten auch zur Ausweisung von Adeligen, Nonnen oder Mönchen führen. Anhörungen und in Augenscheinnahmen, Grenzkontrollen oder die Ausstellung von Passierscheinen werden damit nicht allein als Praktiken der Sicherheitsproduktion fassbar, sondern sie trugen dazu bei, bestehende Differenzen zu manifestieren oder auch zu relativieren. 


\section{Zugehörigkeiten und Grenzziehungen}

Im Sommer 1376 schlossen die Vertreter vierzehn schwäbischer Reichsstädte ein Bündnis miteinander, um sich künftig besser gegen die stetigen Angriffe auf ihre Privilegien und Freiheiten verteidigen zu können (vgl. Ruser 1988, Bd. 2, 2, S. 600 - 601). Der Allianz von kleineren wie größeren Reichsstädten, darunter Ulm und Konstanz, traten schon bald darauf weitere Reichsstädte aus Schwaben, Franken und Bayern bei, so dass der sogenannte Süddeutsche Städtebund in der Mitte der 1380er Jahre rund vierzig Mitglieder zählte (vgl. Rüther 2014; Kreutz 2005; Angermeier 1966). Von Beginn an als militärisches Bündnis konzipiert, kämpften die Städte in wechselnden Konstellationen gegen den umliegenden Adel, bis die gewaltsamen Konflikte im sogenannten 1. Süddeutschen Städtekrieg von 1388/89 eskalierten (vgl. Rüther 2019).

Die Kriege des Spätmittelalters wurden vornehmlich in Form von Kriegszügen geführt: Bewaffnete Truppen zogen durch das gegnerische Territorium und verwüsteten es durch Raub und Brand. Von der Gewalt waren demnach vor allem Nichtkombattanten, die weitgehend ungeschützte Landbevölkerung, betroffen, während die Kämpfer selbst selten in direkte kriegerische Auseinandersetzung mit dem Gegner verwickelt wurden (vgl. Rüther 2009b; Prietzel 2006; Clauss 2006; Nicholson 2004). Und auch die direkten Angriffe auf die Städte, wie die Belagerung Ulms 1376, blieben die Ausnahme und in der Regel ohne Erfolg. Denn zumindest die größeren Städte waren durch ihre Stadtbefestigung und die Verpflichtung aller männlichen Bürger zum Wehrdienst mit eigenen Waffen verhältnismäßig gut geschützt (vgl. Biller 2016; Isenberg/Scholkmann 1997). Es ist zu betonen, dass die Reichsstädte an diesen Kriegen keineswegs nur defensiv teilhatten, sondern diese selbst aktiv und mit eigenen politischen wie finanziellen Interessen betrieben, was wiederum vor allem zu Lasten der Bewohner*innen des städtischen Umlands ging (vgl. Rüther 2020). Daher suchten in Zeiten wachsender Kriegsgefahr mehr und mehr Menschen den Schutz der Stadtmauern und drängten in das Innere der Städte (vgl. Simon-Muscheid 2000). Die städtischen Obrigkeiten reagierten darauf mit einer Reihe von Maßnahmen, um den Zugang und Aufenthalt in der Stadt zu regulieren und zu kontrollieren. Der Schwerpunkt der Analyse wird im Folgenden auf der Sicherheitspolitik des Nürnberger und Regensburger Rates liegen, da hier die Überlieferung der ratsherrlichen Ordnungen und Erlasse besonders dicht ist (vgl. Schubert 2003; Engelke 1995). So erließ der Nürnberger Magistrat zu Beginn des Krieges 1388 ein Mandat, mit dem er strikte Grenzen zwischen den Menschen zog, die zu diesem Zeitpunkt in der Stadt lebten: Alle, die nicht Nürnberger Bürger*in- 
nen waren, hatten die Stadt innerhalb der nächsten zwei Tage zu verlassen, egal ob Mann oder Frau (vgl. Ruser 2005, Bd. 3, 3, S. 2357).

Um das Nürnberger Bürgerrecht zu erwerben, musste man ein Mindestvermögen nachweisen, zudem wurde eine Aufnahmegebühr erhoben (vgl. Schultheiss 1972). Der Erwerb des Bürgerrechts war in Nürnberg, wie in den spätmittelalterlichen Städten üblich, an den Besitz von Waffe und Harnisch gekoppelt. Das geforderte Ausmaß der Bewaffnung war abhängig vom jeweiligen Vermögen des Einzelnen, wie das in Nürnberg geführte „harnasch puch" belegt (Endres Tucher 1864, S. 20; vgl. Zeilinger 2007; Saur 1911). Wer Nürnberger Bürger war, musste im Inneren der Stadt wohnen und dort über ein eigenes Haus verfügen, wodurch das Bürgerrecht zu einem Merkmal sozialer Differenzierung wurde, das sich in der städtischen Topographie niederschlug (vgl. Dirlmeier/ Fuhrmann 2005; Schnelbögl 1982; Piper 1982). Vom Bürgerrecht ausgeschlossen blieben diejenigen, die über keinen eigenen Hausstand verfügten, also Knechte und Mägde, Tagelöhner und andere Angehörige der Unterschichten, die im ausgehenden Mittelalter schätzungsweise ein Drittel der Einwohner*innen Nürnbergs ausmachten (vgl. Gröbner 1993).

Angesichts einer stark wachsenden Bevölkerungszahl in den 1360er und 1370er Jahren und offenbar in Vorbereitung auf den erwarteten Krieg hatte der Rat der Stadt 1382 ein "new gebot“ (Nürnberger Bürgerbücher, S. 85) erlassen, das unterschiedliche Qualitäten des Bürgerrechts festschrieb. Es sollte den Zuzug von Neubürgern auf die nächsten fünf Jahre erleichtern, zugleich teilte es die Nürnberger Einwohnerschaft in drei Gruppen, die durch unterschiedliche Rechte und Pflichten gekennzeichnet waren und in der städtischen Topographie durch unterschiedliche Wohnorte konstituiert wurden (vgl. Schultheiss 1972, S.180-182). Die Grenzen zwischen den Gruppen wurden durch eine Mischung aus sozialem Status, gemessen an ihrer ökonomischen Potenz, und der jeweiligen Funktion im wirtschaftlichen Gefüge der Stadt markiert (vgl. Dirlmeier/ Fuhrmann 2005). Um Bürger Nürnbergs werden zu können, musste man über ein Vermögen von mindestens 200 Florin (Florentiner Goldmünzen) verfügen und eine Aufnahmegebühr von zehn Florin entrichten. Doch nur, wer mit Erlaubnis des Rates ein Haus im Inneren der Stadt, im Stadtviertel St. Sebald oder St. Lorenz im Wert von mindestens 50 Florin erworben hatte, galt als Vollbürger.

Von diesen abgegrenzt wurden zweitens die Handwerksmeister oder Angehörige anderer höher angesehener Berufsgruppen, wie etwa Krämer, Brauer oder auch Maler. Diese mussten eine geringere Aufnahmegebühr zahlen und sich zunächst für fünf Jahre in den Vorstädten ansiedeln, die zu dieser Zeit ummauert wurden (vgl. Schultheiß 1969). Sie wurden als 
Vorstadt- oder Minderbürger in den Neubürgerlisten geführt. Dass zudem der jeweilige Beruf aufgeführt wurde und sie in den Quellen auch „hantwerkspürger" (Schultheiß 1972, S. 181) genannt werden, verweist auf eine auch horizontale Differenzierung der Nürnberger Einwohnerschaft im Rahmen des Bürgerrechts (vgl. Hillebrand 2001). Für den Erwerb eines Hauses im Inneren der Stadt nach Ablauf der Fünfjahresfrist galten strenge Regeln, die einen Kaufpreis von mindestens 20 Florin und die Zustimmung des Rates vorschrieben, wodurch die soziale Differenzierung nach Vermögen in der räumlichen Gliederung der Stadt manifest wurde (vgl. Schultheiß 1965, S. 318).

So mussten auch die Angehörigen der dritten Gruppe, „(sleht) tagwerker" (Schultheiß 1972, S. 181) genannt, in den Vorstädten wohnen, sofern sie nicht im Haushalt eines Bürgers lebten und dort ihren Dienst taten. Sie galten nicht als Bürger*innen, sondern als Einwohner*innen, und ihr Aufenthaltsrecht in der Stadt konnte vom Rat jederzeit wieder zurückgenommen werden (vgl. Nürnberger Bürgerbücher 1974, S. 97-98). Sie waren daher auch von den 1388 erlassenen Geboten zur Ausweisung von Nichtbürgern betroffen, allerdings hatte der Rat der Stadt noch eine weitere Unterscheidung eingeführt: „der burger diener und ehalten, die hie dienen und der burger pawren die herren hynnen haben" (Ruser 2005, Bd. 3, 3, S. 2357) sollte der weitere Aufenthalt in der Stadt trotz des bevorstehenden Krieges weiterhin gestattet sein. Mit der „burger diener" waren Männer gemeint, die zur Unterstützung im Wach- und Kriegsdienst von den Bürgern angeworben wurden oder, wie die „burger pawren“, als Abhängige den Bürgern zu bestimmten Frondiensten verpflichtet waren. „Eh[e]halten“ umfasste hingegen die Gruppe der Dienstboten beiderlei Geschlechts, die in der Regel in den Bürgerhäusern lebten und als zum Haushalt gehörig galten. Ein nachweisbares Dienst- und Abhängigkeitsverhältnis und damit ein gewisses $\mathrm{Ma}$ an sozialer Einbindung wurde in diesen Fällen als hinreichende Voraussetzung angenommen, um als zur Stadtgemeinschaft zugehörig zu gelten, selbst wenn die Betroffenen wie die genannten Bauern im städtischen Umland lebten.

Eine andere große Gruppe, die zwar dauerhaft in der Stadt ansässig war, aber kein Bürgerrecht besaß, waren die „pfaffen, munich, Deutschherren, gaistlich lewt" (Ruser 2005, Bd. 3, 3, S. 2358). Mit sechs Männerklöstern, darunter das erst 1380 gestiftete Kartäuserkloster, zwei Frauenklöstern und der Deutschherrenkommende, einer bedeutenden Niederlassung des Deutschen Ordens, im Inneren der Stadtmauern war von dieser Anordnung eine große Anzahl von Menschen betroffen. Hinzu kam der zu den Stadtpfarrkirchen gehörige Klerus (vgl. Northemann 2011). Alle diese Personen waren in die Gehorsams- und Abhängigkeitsverhältnisse ihres Bistums 
bzw. ihres jeweiligen Ordens eingebunden, was der Aufnahme in das Nürnberger Burgrecht und der Ableistung des Bürgereids prinzipiell entgegenstand (vgl. Möller 1971). Doch nun, angesichts der Gefahr eines Krieges, sollten sie, falls sie weiterhin in Nürnberg bleiben wollten, der Stadt Treue schwören und bei den Heiligen beeiden, „daz sie nichts melden und warnen, daz der stat schedlich sey, mit briefen und boten noch mit in selben“ (Ruser 2005, Bd. 3, 3, S. 2358, vgl. Holenstein 2008). Der Inhalt des von den Klerikern und Ordensleuten zu leistenden Eids verweist darauf, dass der Rat offenbar fürchtete, die Geistlichen, die über die kirchliche Hierarchie oder die jeweilige Ordensstruktur über überregionale Beziehungen verfügten, könnten kriegsrelevante Informationen aus der Stadt herausbringen. Dass Frauen im Mittelalter und bis weit in die frühe Neuzeit nicht als eidesfähig galten, erklärt, warum die Nonnen in der Aufzählung fehlen und die Frauen in einem anderen Kontext und an einer anderen Stelle der Anordnung genannt werden.

Neben der Geistlichkeit gab es auch eine Reihe von Laien, die sich längerfristig in der Stadt aufhielten, aber kein Nürnberger Bürgerrecht besaßen, wie etwa die Kaufleute aus den anderen Städten des Reiches oder Oberitalien. Dieser Gruppe, die in Friedenszeiten einen selbstverständlichen Teil des städtischen Alltags ausmachte, war, wie einzelne Bestimmungen des Satzungsbuchs etwa zum Verbot des Waffentragens deutlich machen, der vorübergehende Aufenthalt in der Stadt erlaubt (vgl. Schultheiß 1965, S. 43). Die grundsätzliche Offenheit der Stadt bildete für Nürnbergs Stellung als überregionaler Handelsort eine notwendige Voraussetzung (vgl. Kießling 2019; Braunstein 1967). Nun begegnete man den „Gästen“ jedoch mit Misstrauen, da ihre Loyalitäten nicht eindeutig geklärt waren. Bereits 1384 hatte der Nürnberger Rat angeordnet, dass alle, die in der Stadt wohnhaft, aber keine Bürger waren, und die „eygen rauch', weib oder kint hie haben" (Schultheiß 1965, S. 306), also einen eigenen Hausstand führten, innerhalb von acht Tagen Nürnberg verlassen sollten und sich auch in Zukunft nicht mehr in Nürnberg niederlassen dürften. Doch nicht allein der längerfristige persönliche Aufenthalt ohne Bürgerrecht wurde sanktioniert, auch der persönliche Besitz von Häusern, Höfen, Stallungen oder Gärten innerhalb der Stadtmauern sollte den Nichtbürgern verwehrt werden. Etwaige Mieter solcher Immobilien waren unter Androhung von Strafe aufgerufen, die Mietzahlungen einzustellen und die Eigentümer innerhalb von vierzehn Tagen zu melden (vgl. Schultheiß 1965, S. 306; Schultheiß 1971, S. 182). Das Misstrauen, das man den Gästen gegenüber hegte, spricht auch aus einer vermutlich zeitgleichen Anordnung, der zufolge "dhein burger noch maister dheinem gast sein tochter nicht gheben" sollte (Schultheiß 1965, S. 262). Voraussetzung, um als Auswärti- 
ger in die Stadt Nürnberg, einheiraten' zu können, war eine Art Leumundszeugnis seiner bisherigen Obrigkeit, dass er ehelich geboren und in „einem guten Wort und leymund herkommen sey“ (ebd.). Die Regelungen verweisen auf das Verlangen des Rates, klare $\mathrm{Zu}$ - und Einordnungen vornehmen zu können, wobei es auch um finanzielle Interessen ging. Nicht allein dem Ausfall von Bürger- oder Steuergeldern wollte man vorbeugen, sondern unklare Zugehörigkeiten führten häufig zu Konflikten um Besitz- und Erbrechte, die dann vor bzw. von den jeweiligen Obrigkeiten ausgetragen werden mussten, was kosten- und zeitaufwendig war.

Dass aber solche rigiden Maßnahmen wie die Ausweisung von Personen ohne Bürgerrecht auch tatsächlich umgesetzt wurden, zeigen Einträge im Acht-, Stadtverbots- und Strafbuch der Stadt Nürnberg aus den Jahren 1381 bis 1403 (vgl. Schultheiß 1960, S. 92-130). So wurde im Juli 1381 einem Hansen von Tryer „die stat verboten 1 jar und funf meyl hindan“ (Schultheiß 1960, S. 94), weil er in der Stadt gewohnt hatte, ohne ihr Bürger zu sein. Der Stadtverweis erfolgte aufgrund einer Klage der Kürschner, er habe unberechtigterweise „unterkauff“ betrieben, sei also als Zwischenhändler aufgetreten, ohne dafür zugelassen worden zu sein. Im gleichen Jahr wurde einer Frau „Zeppfin“ verboten, sich jemals wieder in der Stadt niederzulassen, da sie sieben Jahre lang in Nürnberg gelebt habe, ohne Burgrecht gehabt zu haben. Ihr sollte es aber weiterhin erlaubt sein, "gastesweyse auz- und ein[zu]ziehen" (Schultheiß 1960, S. 97). Dass der Stadtverweis als Strafe sowohl zeitlich wie räumlich differenziert ausgesprochen werden konnte, macht die Bedeutung des Stadtinneren als Sozial- und Schutzraum deutlich. So machte es einen Unterschied, ob man sich der Stadt für ein oder mehrere Jahre nur auf fünf oder neun Meilen nähern durfte, wenn es darum ging, die familiären oder geschäftlichen Beziehungen aufrechtzuerhalten. Zugleich bot das unmittelbare städtische Umland noch einen gewissen Schutz bei feindlichen Angriffen, da es in das städtische Wach- und Verteidigungssystem der Stadt mit eingebunden war. So wird verständlich, dass einzelne Personen versuchten, die Stadt trotz Verbots wieder zu betreten, wie die „Hoerschin“, die einen erneuten Stadtverweis für fünf Jahre und auf fünf Meilen erhielt, da sie trotz eines zuvor ausgesprochenen Verbots in der Stadt angetroffen worden war (Schultheiß 1960, S. 109). Es ist dies einer der wenigen Hinweise darauf, wie die ordnungspolitischen Maßnahmen des Rates von den Betroffenen wahrgenommen wurden bzw. welche Möglichkeiten sie hatten, sich ihnen zu entziehen. Es lässt sich demnach vermuten, dass trotz der wiederholten Anordnungen in den Satzungsbüchern und den unterschiedlichen Sanktionsmaßnahmen zum Zeitpunkt der expliziten Ausweisung der Gäste von 1388 eine ganze Reihe von Personen ohne Bürgerrecht innerhalb der 
Stadtmauern lebte, die jedoch aufgrund ihres unterschiedlichen rechtlichen und ständischen Status auch unterschiedlich behandelt wurden. So hatten die ,fremden' Kaufleute und ihre Familien wie die Geistlichen die Möglichkeit, ihre Treue zur Stadtgemeinschaft mit einem persönlichen Eid zu erklären, um auch während des Krieges in Nürnberg bleiben zu können (vgl. Ruser 2005, Bd. 3, 3, S. 2358).

Diese Option blieb einer anderen Gruppe von Personen ohne Bürgerrecht, die ebenfalls längerfristig in der Stadt lebte, aufgrund ihres sozialen Status verwehrt. Die Anordnung sah vor, dass falls ,iemant hynnen wer, der gern hie belieb und doch nicht burger wer, als junkfrawen, pegein, armlewt", eine Dreierkommission aus dem Kreis des Rates darüber befinden sollte, ob diese oder dieser der Stadt schädlich sein könnten, und ihnen andernfalls ein Bleiberecht erteilen. An die Stelle einer Selbstverpflichtung durch einen persönlichen Eid trat hier also die Fremdeinschätzung durch die Obrigkeit. Dass von dieser Regelung vor allem Frauen betroffen waren, wird durch die explizite Nennung der "Jungfrauen“ und „Beginen“ deutlich. Dabei lässt sich aufgrund der begrenzten Quellenüberlieferung nicht eindeutig klären, ob mit den „Jungfrauen“ die Nonnen in der Stadt gemeint sind, wofür die gleichzeitige Nennung der Beginen als Semireligiose sprechen würde. Doch als Jungfrauen wurden im Mittelalter auch die jüngeren und unverheirateten Dienstmägde und Dienerinnen bezeichnet, die eher in der Nähe der "armlewt“ zu sehen wären. Möglicherweise sollten mit den "Jungfrauen“ aber beide Gruppen angesprochen, denn wenngleich in der sozialen Hierarchie der Stadt sehr unterschiedlich zu verorten - die Ordensfrauen waren wie die Dienstmädchen als weibliche Personen von der Möglichkeit der persönlichen Eidesleistung ausgeschlossen und damit dem Urteil der Ratsherren unterworfen.

Die Verordnung des Nürnberger Rats von 1388 schloss nicht nur einzelne Personengruppen explizit aus der Stadtgemeinschaft aus, sondern bekrättigte zugleich die innere Hierarchie der Stadtgemeinde, indem sie Zuordnungen und Abhängigkeitsverhältnisse abfragte und den jeweiligen Grad der Zugehörigkeit durch verschiedene Praktiken der Beglaubigung wie Eid und Inaugenscheinnahme markierte. Die Unterscheidungen, die dabei im Hinblick auf die Sicherheit der Stadt getroffen wurden, referenzierten in erster Linie auf den sozialen Status und die jeweilige Einbindung in soziale Figurationen. So konnte der Magd eines Ratsherrn aufgrund ihrer Zugehörigkeit zu dessen Haushalt der Aufenthalt in der Stadt weiterhin gestattet sein, während ein Mitglied des Deutschen Ordens aufgrund seiner auswärtigen Verbindungen und Loyalitäten als potentiell unsicher galt und die Stadt verlassen musste. Eine weibliche Angehörige des Dominikanerordens aus dem Nürnberger Katharinenkloster hingegen 
hatte nicht wie ihr Ordensbruder aus dem benachbarten Nürnberger Dominikanerkloster die Möglichkeit, ihre Treue zur Stadt mit einem persönlichen Eid zu bekräftigen, sondern war in diesem Fall auf die Fürsprache und das Urteil männlicher Personen angewiesen. Die Sicherheitsmaßnahmen des städtischen Rates zeigen die grundsätzliche Bedeutung der Intersektionalität für das spätmittelalterliche ständische Denken, in dem die Stellung des einzelnen maßgeblich von der Zugehörigkeit zu einer sozialen Gruppe oder Kooperation geprägt war. Zugleich wurde aber auch immer wieder nach Geschlecht, religiöser Zugehörigkeit und sozialem Status differenziert, so dass sich je nach Situation und Perspektive wechselnde Zuordnungen ergeben konnten (vgl. Bähr/Kühnel 2018; Oexle 1998).

\section{Ausgrenzung und Einverleibung}

Eine andere Gruppe, deren Aufenthalt in der Stadt und Zugehörigkeit zur Stadtgemeinschaft im Spätmittelalter prekär war, bildeten die Jüdinnen und Juden. Die überwiegende Zahl der im schwäbischen Städtebund zusammengeschlossenen Reichsstädte hatte ein eigenes ,Judenbürgerrecht', was allerdings wenig über die tatsächliche rechtliche und soziale Stellung in den jeweiligen Stadtgemeinden aussagt (vgl. Gilomen 2002, S. 127; Darman 2009; Scholl 2012). Nürnberg hatte bis zur Ermordung und Vertreibung der Juden 1349 eine der größten jüdischen Gemeinden in Europa mit vermutlich über 1.000 Personen; und auch wenn die Zahl der in Nürnberg lebenden Juden in der zweiten Hälfte des 14. Jahrhunderts weit dahinter zurückblieb, war der Rat an der Neuaufnahme und Ansiedlung von Juden in der Stadt interessiert (vgl. Nürnberger Bürgerbücher 1974, S. 81-84; Müller 1968). 1360 hatte er vom Kaiser den Judenschutz übernommen, was der Stadt erhebliche Einnahmen durch die Erhebung von Schutzgeldern, hohe Aufnahmegebühren und die Erhebung der Judensteuer brachte (vgl. Müller, S. 62, Demel 2011, S. 54-58). Doch bereits zehn Jahre zuvor hatte Karl IV. die Nürnberger jüdische Gemeinde aus seinem kaiserlichen Schutz genommen und dem Rat versichert, dass er keine Entschädigungsleistungen fordern würde, sollten die Nürnberger Bürger die Juden in irgendeiner Form schädigen (vgl. Hegel 1864, S. 332). Das Privileg war Teil der Ausgleichsverhandlungen nach einem innerstädtischen Aufruhr 1348, an dem die Jüdinnen und Juden in Nürnberg keinerlei Anteil hatten (vgl. Schubert 2009). Doch begründet wurde das weitreichende Zugeständnis, das die jüdische Gemeinde vollkommen schutzlos machte, mit einer Gefährdung der Nürnberger Bürger: „wanne die juden zu Nurenberg unser camerknecht itzunt in manigerley vehe des gemainen volkes 
und auch die burger in der stat irs leibs und guout, die weil die juden in der stat seint nicht sicher seint" (Hegel, 1864, S. 323). Die darauffolgende Ermordung und Vertreibung der Juden aus Nürnberg, die Zerstörung der Synagoge und die vollständige Enteignung des jüdischen Besitzes kann mithin als Ergebnis eines erfolgreichen Versicherheitlichungsprozesses gesehen werden, an dessen Ende die Umkehr des Verhältnisses von Schutz und Gefahr, von Gefährdeten und Gefährdern stand (vgl. Waever 1995; Buzan u. a. 1998). Die spätmittelalterliche Rechtskonstruktion der kaiserlichen Kammerknechtschaft stellte die Juden im Reich unter den besonderen Schutz des Kaisers, der damit zugleich den exklusiven Anspruch auf den Einzug der besonderen Judensteuer erhielt (vgl. Battenberg 1987). Doch war es gerade diese Kopplung, die den exklusiven Schutz zu einer besonderen Gefahr für die Juden im Reich werden ließ, wenn der König die mit dem "Judenregal“ verbundenen Einkünfte verpfändete, verschenkte oder vorübergehend auf sie verzichtete (vgl. Müller 2004, S. 63-69). Das verbindet die in den Jahren 1348 und 1349 reichsweit erfolgte Ermordung und Vertreibung der Juden, die gemeinhin vor dem Hintergrund der Pestwelle gesehen wird (vgl. Hawerkamp 1981). Ermöglicht und vermutlich befördert wurden sie jedoch wie in Nürnberg durch entsprechende Privilegien Karls IV., der die Verpfändung der Judensteuer wie auch die Erlaubnis zur Ausplünderung und Vertreibung der Juden wiederholt als Verhandlungsmasse bei der Anerkennung seiner königlichen Stellung nutzte (vgl. die Beispiele bei Müller/Weber 2016). Auch wenn sein Anteil an den Pogromen in der Forschung umstritten ist, stellte er sich der zunehmenden „Kriminalisierung der Juden“ (Müller 2004, S. 96) um die Mitte des 14. Jahrhunderts, wie sie auch aus dem Privileg für Nürnberg spricht, nicht entgegen. Diese Deutung wird noch verstärkt durch die im Wortlaut der Urkunde unmittelbar folgende Erlaubnis an den Rat, jeden aus der Stadt zu verweisen, wenn er den Verdacht hege, dass "der der stat nicht fueglich were" (Hegel, 1864, S. 332).

Das Dokument spiegelt mittelalterliche Praktiken der Sicherheitserzeugung, die darauf zielten, einen sicheren und gewissermaßen ,reinen' Raum zu schaffen, indem Elemente der Verunsicherung abgetrennt und ausgesondert wurden. Das lässt sich im besonderen Maße am Schicksal des alten jüdischen Viertels in Nürnberg ablesen, das im Zentrum der Stadt an der Stelle des heutigen Marktes gelegen hatte (vgl. Kohn 1978). Die Häuser und Grundstücke in begehrter Lage hatte Karl IV. in Erwartung des von ihm erlaubten ,Pogroms` bereits im Juni 1349 an hochrangige Fürsten als Belohnung für erwiesene Dienste verteilt. So sollte Ludwig von Brandenburg drei "der besten Juden haueser“ erhalten, „wann die juden da selbes nu nehst werden geslagen“ (Constitutiones, Bd. 9, S. 298). Dem Bischof 
von Bamberg hatte er wenige Tage zuvor eine jährliche Zahlung von 1.100 Haller aus seinen Einkünften aus der Nürnberger jüdischen Gemeinde versprochen. Sollte diese jedoch die Stadt verlassen oder würde sie vertrieben, bevor die Schuld von insgesamt 7.000 Haller bezahlt wäre, würde der Bischof zusammen mit den Nürnberger Burggrafen je die Hälfte des gesamten jüdischen Besitzes in der Stadt erhalten (vgl. Constitutiones, Bd. 9, S. 289-290). Wenn damit die Ermordung und Vertreibung der Juden in den Jahren 1348/1349, die in nahezu allen fränkischen und schwäbischen Städten sowie in den Bayrischen Herzogtümern vollzogen wurde, vornehmlich durch finanzielle Interessen motiviert erscheint, so zielte sie in Nürnberg zugleich auf eine Umgestaltung der sozialen Topographie im Innersten der Stadt. In der sogenannten Markturkunde vom 16. November 1348 erlaubte Karl IV. Rat und Bürgern der Stadt, mehrere der Häuser im jüdischen Besitz, deren Lage genau bezeichnet wird, abzubrechen, um an dieser Stelle einen Markt zu errichten, „dar in der selben stat kein grozzer platz nicht enist, dar an die leut gemeinlichen an gedienge kaufen und vorkaufen muegen“ (Constitutiones Bd. 9, S. 418). Aus der „judenschul“ hingegen sollte man nach dem Willen des Kaisers „machen eine kirchen in sant Marien ere unser frawen“. Wenige Tage darauf übereignete er dem Ratsherrn Ulrich Stromer für seine Dienste das Haus des Isaak von Schehslitz und gebot Rat und Bürgern der Stadt, alles zu tun, damit Stromer es auch in Besitz nehmen könne (vgl. Constitutiones Bd. 9, S. 481-82). Wenige Wochen später, am 6. Dezember 1349, ermordeten die Nürnberger Bürger vermutlich 562 jüdische Mitbürger und vertrieben die übrigen aus der Stadt. Ihre Häuser wurden von den Angehörigen der städtischen Oberschicht in Besitz genommen oder abgerissen (vgl. Schultheiß 1964, S. 241 und 243). Die ehemals jüdische Mitte der Stadt wurde gleichermaßen materiell überformt wie symbolisch überschrieben: Ulrich Stromer nannte sein neues Anwesen „Zur goldenen Rose“, und ein Teil des bekannten Patriziergeschlechts führte diesen Zusatz künttig sogar in ihrem Namen (vgl. Fleischmann 2008, S. 941-972). Die Synagoge und der jüdische Friedhof wurden zerstört; die Grabsteine zum Bau verwendet, etwa als Stufen für den 1352 neu errichteten Südturm der St. Lorenzkirche (vgl. Müller 1968, S. 32). Ähnliche Praktiken der Einverleibung sind auch aus anderen fränkischen Städten überliefert, wie etwa aus Würzburg, wo die Grabsteine in Bürgerhäusern verbaut oder zur Erneuerung der Stadtmauer verwendet wurden (vgl. Müller 2011). Wie von Karl IV. angeordnet, wurde über der ehemaligen Synagoge die Nürnberger Frauenkirche errichtet, die 1352 erstmals urkundlich erwähnt und Mitte der 1360er Jahre fertiggestellt wurde. 
$\mathrm{Zu}$ dieser Zeit gelang es dem Rat auch, vom König den Judenschutz zu erhalten und erneut Juden in Nürnberg mit eigenem Judenbürgerrecht anzusiedeln (vgl. Müller 1968, S. 62; Nürnberger Bürgerbücher 1974, S. 84). Doch blieb deren räumliche Situation nach der Zerstörung des alten jüdischen Viertels offenbar zunächst prekär; so beklagten sie sich vor dem Nürnberger Rat, „daz sie hie niht wohl herberg" hätten (Schultheiß 1965, S. 320). Die jüdische Gemeinde erhielt daraufhin 1383 die Erlaubnis, im östlichen Teil der Stadt ein geschlossenes Grundstück bzw. eine Reihe von Häuser zu erwerben, für die sie jedoch eine Art Grunderwerbsteuer von 12 Prozent zu entrichten hatten.

Wenn die Juden im Rahmen der Maßnahmen zur Ausweisung der Gäste im unmittelbaren Vorfeld des Krieges nicht eigens adressiert wurden, liegt der Grund hierfür zum einen darin, dass der Aufenthalt von auswärtigen Juden ohne Nürnberger Burgrecht ohnehin streng reglementiert und zeitlich begrenzt war (vgl. Schultheiß 1965, S. 279-280). Zum anderen waren die Juden für die Kriegsfinanzierung des schwäbischen Städtebundes von großer Bedeutung, so dass ihre Anwesenheit in der Stadt nahezu unverzichtbar war. Als es 1384 in Windsheim, Weißenburg und Nördlingen erneut im Kontext von städtischen Unruhen zu Ausschreitungen und der Ermordung von Juden kam, griff der Städtebund ein und verurteilte die Verantwortlichen scharf (vgl. Scholl 2012, S. 120-122). Doch schon im darauffolgenden Jahr einigten die Reichsstädte sich im Zuge der Annäherung an König Wenzel auf die sogenannte Judenschuldentilgung, die faktisch einer Enteignung der jüdischen Bürger in 38 Städten des schwäbischen Bundes gleichkam. In einer konzertierten Aktion wurden am 16. Juni 1385 alle jüdischen Bürger verhaftet und zur Herausgabe aller ihrer Schuldscheine und Pfänder gezwungen (vgl. Holtz 1993, S. 99-101). Den Gläubigern, unter ihnen neben den Bürgern und Stadträten auch zahlreiche Adelige, wurde pauschal ein Viertel der Schulden erlassen, der größte Teil der Restschuld wurde von den Stadträten einbehalten, nachdem König Wenzel - wie schon sein Vater zuvor - ihnen gestattet hatte, bis zum 2. Februar 1388 beliebigen Gewinn aus den Juden zu ziehen. Mochten die Angehörigen der jüdischen Gemeinden ebenso wie viele andere soziale Gruppen angesichts einer erhöhten Kriegsgefahr Sicherheit im Inneren der Städte suchen, so boten die Stadtmauern ihnen im ausgehenden 14. Jahrhundert nur bedingt Schutz. Denn zugleich markierten diese den Geltungsraum der Willkür des städtischen Rates, innerhalb dessen er für sich weitreichende Zugriffsrechte auf Leib und Leben der jüdischen Bürger*innen reklamierte. 
Während die Obrigkeiten auf der einen Seite versuchten, die städtische Gemeinschaft auf einen eigentlichen Kern zurückzuführen, indem sie Personen mit ungeklärtem oder prekärem Status auswiesen, waren sie auf der anderen Seite darum bemüht, die Lasten des Krieges auf möglichst viele zu verteilen. So hatte der Rat der Stadt Konstanz unmittelbar nach der Gründung des städtischen Bundes im Sommer 1376 verfügt, dass es für die nächsten vier Jahre keinem seiner Bürger erlaubt sein sollte, die Stadtgemeinschaft wegen des Bündnisses und den damit verbundenen Verpflichtungen zu verlassen (vgl. Ruser 1988, Bd. 2, 2, S. 607-608). Der Magistrat der Stadt Regensburg, der 1381 dem süddeutschen Städtebund beigetreten war, verlangte 1386 von seinen Bürgern einen zusätzlichen Eid, mit dem sie geloben sollten, „das ir des Kriegs trewlich bi ein ander beleibt mit leib vnd gut als lang der wert vnd an ein ander geraten vnd behelffen seit und gehorsam seit" (Engelke 1995, S. 223-224; zum Beitritt Regensburgs, vgl. Ruser 2005, Bd. 3, 2, S. 1452-1453). Die Eidesformel richtete sich sowohl in die Vertikale mit der Verpflichtung zum Gehorsam gegenüber dem Rat, was insbesondere auf den Kriegszügen ein immer wiederkehrendes Problem darstellte, als auch in die Horizontale mit der Verpflichtung zur gegenseitigen Hilfe der Bürger untereinander (vgl. Rüther 2020, S. 31-33). Auch in Nürnberg sollten die im Zuge des neugestalteten Bürgerrechts von 1382 formulierten Eidesformeln die Bürger und Einwohner zur Treue gegenüber dem Rat und Gehorsam gegenüber ihren jeweiligen Hauptleuten verpflichten. Auffallend ist, dass in den Eidesformeln, vom Eid für die Neubürger bis hin zum Eid der Tagwerker, die Verpflichtung, Schaden von der Stadt abzuwenden, einen besonderen Stellenwert einnahm. So sollten alle Bürger und Einwohner dem Rat melden, wenn sie von etwas wüssten, „was wider den rat und wider die stat wer“, worunter auch Nachrichten von einem „zerwernuezz oder aufleuf“ fallen sollten (Nürnberger Bürgerbücher, S. 96-98).

Trotz einer möglichen Gefährdung im Inneren erlaubte der Regensburger Rat 1386 jedoch denjenigen die Rückkehr, die aufgrund einzelner Vergehen der Stadt verwiesen worden waren, sofern es sich nicht um schwerwiegende Verbrechen wie Mord oder Mordbrennerei handelte. Dass Frauen von dieser Amnestie ausgeschlossen blieben, macht deutlich, dass es dem Magistrat mit dieser Maßnahme wohl in erster Linie darum ging, die städtische Kampfkraft zu verstärken (vgl. Engelke 1985, S. 223, Nr. 304). Die Grenzziehungen richteten sich mithin nach der Funktion und dem möglichen Nutzen im Hinblick auf den bevorstehenden Krieg. Das zeigt auch der zeitgleiche Erlass, mit dem allen Männern das Spielen in der 
Stadt erlaubt war, obwohl die obrigkeitliche Reglementierung von Würfelund Kartenspielen bis hin zum vollständigen Verbot im Spätmittelalter eher der Regelfall war (vgl. Schultheiß 1965, S. 255).

Ein weiteres Mittel, um sich militärisch zu verstärken, war die Aufnahme von Adeligen aus dem Umland als Pfahl- oder Ausbürger, die ohne die Verpflichtung zur Residenz in der Stadt das Bürgerrecht erhielten (vgl. Neubürgerlisten 1974, S.73-76; Marchal 2002; Blickle 2006). Diese Praxis war im Spätmittelalter umstritten und in der Goldenen Bulle sogar explizit verboten worden, denn sie eröffnete vor allem dem niederen Adel die Möglichkeit, sich aus bestehenden Herrschafts- und Abhängigkeitsverhältnissen zu lösen und in eine relativ freie Beziehung zur Stadt zu treten, da in der Regel keine Steuerleistungen erbracht werden mussten. Nürnberg nahm im unmittelbaren Vorfeld des Krieges zwanzig fränkische Adelige für einen begrenzten Zeitraum als Bürger auf, um sich auf diese Weise ihre Unterstützung im Krieg durch persönlichen Kriegsdienst sowie den $\mathrm{Zu}$ gang zu den Schlössern im Umland als strategisch wichtige Stützpunkte zu sichern (vgl. Neubürgerlisten 1974, S. 75; Rüther 2010). Der Rat sah in der zeitweisen Aufnahme in das Bürgerrecht und dem damit verbundenen Bürgereid offenbar eine Möglichkeit, die Adeligen nachhaltiger an die Stadt zu binden als durch den Abschluss eines bloßen Soldvertrags. Zugleich war Nürnberg wie die anderen Städte auch darauf angewiesen, durch die Anwerbung von adeligen wie nichtadeligen Söldnern die eigenen Truppen zu verstärken (vgl. Rogger/Schmid-Keeling 2019; Rüther 2019).

Dem Wunsch nach einer klaren Abgrenzung oder gar Abschottung der Stadt nach außen stand somit die Notwendigkeit gegenüber, weiterhin die Zirkulation von Personen, Waren und Informationen zuzulassen, um die für die Kriegführung notwendigen Ressourcen sicherzustellen. Die Stadttore wurden zu neuralgischen Punkten zwischen dem Innen und Außen der Stadt und deshalb strengstens kontrolliert. Laut einer Anordnung des Regensburger Rates vom Beginn des Jahres 1388 hatten die Torwächter jeden, der herein oder heraus wollte, zu befragen, wer er sei und was er vorhabe (vgl. Ruser 1985, Bd. 3, 3, S. 2208). Doch die persönliche Auskunft allein genügte nicht, vielmehr benötigte man zum Betreten oder Verlassen der Stadt nun einen Passierschein, Pollitie (politen) genannt. Diese Regelung galt auch und explizit für die Geistlichen und Klosterangehörigen der Stadt, die im Gegensatz zu den auswärtigen, als fremd wahrgenommenen Klerikern, Mönchen und Nonnen im Schutz der Stadtmauern verbleiben durften. Offenbar befürchteten die Regensburger Ratsherren, dass diese Gruppen kriegswichtige Informationen nach außen tragen könnten, weshalb die Torwächter Anweisung hatten, niemanden von ihnen aus der 
Stadt hinauszulassen, es sei denn sie hätten „eine gewizze kuntschaft" vom Rat und eine Pollitie (Ruser 1985, Bd. 3, 3, S. 2208). Man drohte den Geistlichen bei Übertretung dieses Gebots sogar mit körperlicher Bestrafung: „Wer aber des nicht taet, dem will man all sein hab nemmen vnd dannoch mit seinem leib in eine pezzerung schikchen, die im gar zu swaer wirt" (ebd.). Die Anordnung deutet zugleich darauf hin, dass sich der Rat die zum Teil überregionalen Kontakte der geistlichen Personen auch zu Nutzen machte, um Nachrichten zu übermitteln und Informationen zu erhalten.

Die Passierscheine, die man nicht nur zum Einlass, sondern auch zum Verlassen der Stadt benötigte, durften in Regensburg nur von einer Person, dem Ratsherrn Thomas Sittauer, ausgestellt werden, womit die Echtheit des Dokuments gewährleistet und zugleich ihre Anzahl kontrolliert werden konnte (vgl. Engelke 1985, S. 444). Auch in Nürnberg suchte man die Personenströme durch ein solches Papier zu regulieren. Wer während des Krieges eine auswärtige, also fremde Person beherbergte, der oder die keine vom Rat ausgegebene Pollitie besaß, hatte harte Sanktionen zu befürchten (vgl. Ruser 1988, Bd. 3, 3, S. 2379-2380).

Doch zusätzlich zu diesen Praktiken der Beglaubigung durch persönlichen Eid und die Ausstellung von individuellen Dokumenten waren die städtischen Obrigkeiten bei der Identifikation einzelner Personen auf die persönliche Vertrautheit in den Nachbarschaften angewiesen. In Nürnberg hatten die jeweiligen Stadtviertel, wie bereits gesehen, für die Einteilung und Einordnung der Stadtbewohner eine besondere Bedeutung und bildeten die Grundlage für ein besonderes System der räumlichen Kontrolle. So waren den jeweiligen sozialen Gruppen von der patrizischen Oberschicht über die Handwerker bis zu den Juden bestimmte, räumlich klar umgrenzte Wohnorte zugewiesen. Jedes Viertel unterstand einem Viertelmeister, dem wiederum zur Aufsicht der einzelnen Straßenzüge Gassenhauptleute unterstellt waren (vgl. Schall 1971; S. 61-65). Diese räumlich-hierarchische Gliederung diente nicht nur der Organisation des Wach- und Kriegsdienstes, sondern ermöglichte den Obrigkeiten auch eine kleinteilige Kontrolle des städtischen Raums und der darin lebenden Personen. So sollten nach dem Ablauf der Zweitagesfrist, innerhalb derer die Nichtbürger 1388 die Stadt zu verlassen hatten, die Viertelmeister und Gassenhauptleute von Haus zu Haus gehen und die Bürger mit Verweis auf ihre geleisteten Eide befragen, ob sie von Personen ohne Bürgerrecht wüssten, die sich noch in der Stadt aufhielten (vgl. Ruser, 2005, Bd. 3, 3, S. 2357-58). Mit der Anordnung wurden die Bürger Nürnbergs unter Androhung von Strafe in ein System der wechselseitigen Kontrolle eingebunden, zugleich wurde auf 
diese Weise die durch ratsherrliche Erlasse vorgenommene Binnendifferenzierung implementiert.

Die einzelnen Wohnhäuser und die Zugehörigkeit zu einem bestimmten Haushalt über Verwandtschafts- oder Dienstbeziehungen bildeten nach den Stadtvierteln und Gassen die Kernelemente des städtischen Raums als Sicherheitsraum (vgl. Eibach 2015). So wie als unsicher geltende Personengruppen nicht im Inneren der Stadtmauern verbleiben sollten, durften sich auch in den Bürger- und Mietshäusern nur die Menschen kurz- oder langfristig aufhalten, deren Identität und Zugehörigkeit geklärt und bezeugt werden konnte. Nach einer in Regensburg 1388 vorgenommenen Verschärfung des Gebots zur Ausweisung fremder oder gefährlicher Personen durfte Bettlern nur mehr an der Haustüre Almosen gegeben werden, ein Einlass in die Wohnungen war bei Strafe verboten (vgl. Ruser 2005, Bd. 3, 3, S. 2178-2179). Die Sicherheit und Kontrolle der Mitglieder eines jeden Haushalts lagen in der Verantwortung des jeweiligen Hausherrn, doch wurden diese durch Rundgänge und in Augenscheinnahmen der Gassenhauptleute selbst wieder kontrolliert.

Die umfassenden Eingriffe in das soziale Leben und Gefüge der Stadtbewohner angesichts einer drohenden Kriegs- und Krisensituation konnten nicht ohne Widerstand und deviantes Verhalten bleiben. Insbesondere im Hinblick auf die geistlichen Personen wird es zu Problemen gekommen sein, denn gerade unter diesen hatten die Stadtbewohner besondere Vertrauenspersonen, zudem oft auch enge Verwandte. So finden sich, wenn auch nur vereinzelt, Hinweise auf abwehrende Reaktionen der Stadtbewohner auf die von den Obrigkeiten vorgenommen Einteilungen und Differenzierungen, wie etwa die Verurteilung des Regensburger Bürgers Pesel Schnizzer zu einer Leibesstrafe im Juli 1388, dem vorgeworfen wurde, dass er während des Krieges drei Geistliche in seinem Haus beherbergt hatte, deren Aufenthalt im Schutz der Stadtmauern nicht erlaubt gewesen war (vgl. Engelke 1985, S. 439f).

\section{Zusammenfassung}

Die spätmittelalterlichen Stadtgemeinden werden gemeinhin als face-toface Gesellschaften beschrieben, deren soziale Ordnung wesentlich durch gegenseitiges Kennen- und Anerkennen stabilisiert wurde. Doch diese Grundlage wurde durch die ratsherrlichen Anordnungen in Kriegszeiten in Frage gestellt. Der soziale Status jedes Einzelnen und jeder Einzelnen wurde erneut abgefragt, durch Eide bestätigt und musste schließlich auch durch schriftliche Dokumente nachgewiesen werden. Zu betonen ist, dass 
die Infragestellung des jeweiligen Status nicht durch eine Veränderung im Verhalten des einzelnen Individuums oder einer bestimmten Personengruppe motiviert war, sondern durch eine veränderte Sicherheitssituation.

Die Maßnahmen, die die städtischen Obrigkeiten ergriffen, um angesichts eines drohenden Krieges die Sicherheit der Stadt zu gewährleisten, zeugen von der Spannung zwischen dem Bestreben, die Zahl der Menschen innerhalb der Stadtmauern auf einen Kreis vertrauenswürdiger Menschen zu begrenzen, und der Notwendigkeit, auf eine hohe Anzahl an Personen für die Kriegführung zurückgreifen zu können, von den adligen Kämpfern und Söldnern über Händler*innen bis hin zu den Juden als Geldgebern oder Geistlichen als Informanten oder Vermittlern. Angesichts einer erhöhten Kriegsgefahr fürchteten sie zu allererst die Auflösung ihrer Gemeinden, ein Auseinanderdriften von Personen und Gütern, eine Einmischung und Vermischung mit anderen Kräften und Akteuren. Wie von der Forschung als typisch für mittelalterliche Herrschaften beschrieben, reagierten die Stadträte darauf mit einer Intensivierung personeller Bindungen. Mit zusätzlichen Eiden wurde die Zugehörigkeit jedes einzelnen zur Stadtgemeinde bekräftigt und Gehorsam gegenüber den Anordnungen des Rates eingeschärft.

Die ratsherrlichen Erlasse zur inneren Ordnung der Stadt zielten auf die Kontrolle des sozialen wie physischen Orts eines jeden einzelnen Bewohners, die zumindest für die Dauer des Krieges festgeschrieben werden sollte. Die städtischen Machtträger folgten dabei der Fiktion eines ursprünglichen und eigentlichen Kerns der Stadt, der durch die Beseitigung von Uneindeutigkeiten wiederhergestellt werden konnte. Doch ist den zahlreichen Anordnungen der städtischen Obrigkeiten in Kriegszeiten gemeinsam, dass sie trotz dieser Vorstellung eines geschlossenen Ganzen, immer nur auf einzelne Teile, Personen, Güter und Handlungen zielten. Sie waren demnach eher dazu geeignet, die Segmentierung der Stadtgemeinde zu markieren als ihre Geschlossenheit zu forcieren.

Die vorgenommenen Differenzierungen referierten auf die vor der Krisensituation gültigen Marker wie die finanzielle und soziale Stellung als Bürger*in oder Einwohner*in, die ständische Zugehörigkeit, die Religion und das Geschlecht. Doch wurden diese Kategorien mit Bezug auf die Sicherheit der Stadt einer neuen Wertung unterzogen, indem danach gefragt wurde, in welchem Maße die jeweilige Person oder Personengruppe der Stadt durch ihre Beziehungen und Fähigkeiten von Nutzen sein oder Schaden zufügen könnten. Man ist versucht, in diesem Zusammenhang von Ansätzen einer funktionalen Differenzierung im Rahmen eines grundsätzlich ständischen Settings zu sprechen, doch waren diese Formen der Grenzziehungen nur vorübergehend und auf die historisch spezifische 
Situation des Krieges ausgerichtet. Sie sollten daher nicht als Ausdruck langfristiger historischer Modernisierungsprozesse von einer ständisch stratifizierten hin zu einer funktional ausgerichteten Gesellschaft verstanden werden, die für die Städte des ausgehenden Mittelalters in dieser Pauschalität nicht nachweisbar sind. Ich möchte daher vorschlagen, von einer pragmatischen und situationsbezogenen Differenzierung als Repertoire der Versicherheitlichung zu sprechen.

\section{Literatur}

\section{Quellen}

Constitutiones et acta publica imperatorum et regum (1349) (1983). Bd. 9. Nachdruck. Wiesbaden: Harrassowitz.

Endres Tuchers Memorial 1421 bis 1440 (1864). In: Die Chroniken der fränkischen Städte. Nürnberg. Bd. 2. Leipzig: Hirzel, S. 3-53.

Engelke, Thomas (1995): Eyn grosz alts Statpuech. Das „Gelbe Stadtbuch“ der Stadt Regensburg. Forschungen und Edition. Regensburg: Univ.-Verl.

Hegel, Karl (Hrsg.) (1862): Beilagen. In: Die Chroniken der fränkischen Städte. Nürnberg Bd. 3, Leipzig: Hirzel, S. 307-336.

Die Nürnberger Bürgerbücher (1974): I. Die Pergamentenen Neubürgerlisten 1302-1448, mit einer Einführung in die mittelalterlichen Quellen zur Bevölkerungs- und Sozialgeschichte Nürnbergs. Nürnberg: Selbstverlag des Stadtrats Nürnberg.

Ruser, Konrad (1988): Die Urkunden und Akten der oberdeutschen Städtebünde vom 13. Jahrhundert bis 1549. Bd. 2, 1-2: Städte- und Landfriedensbündnisse von 1347 bis 1380. Göttingen: Vandenhoeck und Ruprecht.

Ruser, Konrad (2005): Die Urkunden und Akten der oberdeutschen Städtebünde. Oberdeutsche und schweizerische Städte- und Landfriedensbündnisse von 1381 bis 1389, Bd. 3, 1-3. Göttingen: Vandenhoeck und Ruprecht.

Schultheiss, Werner (1960): Die Acht-, Verbots- und Fehdebücher Nürnbergs von 1285-1400. Mit einer Einführung in die Rechts- und Sozialgeschichte und das Kanzlei- und Urkundenwesen Nürnbergs im 13. und 14. Jahrhundert. Nürnberg: Selbstverlag des Stadtrats Nürnberg.

Schultheiß, Werner (1965): Satzungsbücher und Satzungen der Reichsstadt Nürnberg aus dem 14. Jahrhundert. Lfg. 1: Einleitung (1. Teil), Abdruck der Texte, Personen- u. Ortsregister. Nürnberg: Selbstverlag des Stadtrats 


\section{Sekundärliteratur}

Angermeier, Heinz (1966): Königtum und Landfriede im deutschen Spätmittelalter. München: Beck.

Baeriswyl, Armand (2018): Torturm und Zinnenkranz. Die Stadtbefestigung als Mittel der kommunalen Selbstinszenierung im Mittelalter und in der frühen Neuzeit. In: Stercken, Martina/ Hesse, Christian (Hrsg.): Kommunale Selbstinszenierung. Städtische Konstellationen zwischen Mittelalter und Neuzeit. Zürich: Chronos, S. 253-276.

Bähr, Matthias/Kühnel, Florian (Hrsg.) (2018): Verschränkte Ungleichheit. Praktiken der Intersektionalität in der Frühen Neuzeit. Berlin: Duncker und Humblot.

Battenberg; Friedrich (1987): Des Königs Kammerknechte. In: Historische Zeitschrift 245, S. 545-599.

Bauernfeind, Walter (2019): Vom Judenviertel zur neuen Stadtmitte: Topographie Nürnbergs vor und nach dem Judenpogrom unter Karl IV. In: Lehner, Julia (Hrsg.): Politik. Macht. Kultur. Nürnberg und Lauf unter Kaiser Karl IV. und seinen Nachfolgern. Nürnberg: Stadt Nürnberg, S. 18-45.

Biller, Thomas (2016): Die mittelalterlichen Stadtbefestigungen im deutschsprachigen Raum. Ein Handbuch, 2 Bde. Darmstadt: WBG.

Blickle, Peter: Pfalbürger schwäbischer Reichsstädte. Ein Beitrag zur Konstruktion der Leibeigenschaft. In: Burkhardt, Johannes (Hrsg.): Geschichte in Räumen. Festschrift für Rolf Kießling zum 65. Geburtstag. Konstanz 2006, S. 51-72.

Braunstein, Philippe (1967): Wirtschaftliche Beziehungen zwischen Nürnberg und Italien im Spätmittelalter. In: Beiträge zur Wirtschaftsgeschichte Nürnbergs, hrsg. vom Stadtarchiv Nürnberg. Nürnberg, Selbstverlag des Stadtrats, Bd. 1, S. 377-406.

Buzan, Barry/Waever, Ole/Wilde, Jaap de (1998): Security. A New Framework for Analysis. Boulder, CO: Lynne Rienner.

Clauss, Martin (2006): Aujourd'huy toutes les guerres sont contre les povres gens. Gewalt gegen Nichtkombattannten als Mittel der Kriegsführung im Hundertjährigen Krieg. In: Saeculum 57, S. 77-99.

Darman, Ashkira (2009): Stewren, raisen, wachen, schenken. Steuern und Rechte jüdischer Bürger im Vergleich mit christlichen rechtlichen Gruppen und im Rahmen des städtischen Finanzhaushaltes in Reichsstädten im Südwesten des Reiches (1350-1500). Diss. Zürich Online verfügbar unter https://www.zora.uzh .ch/id/eprint/163842/.

Demel, Michael (2011): Gebrochene Normalität. Die staatskirchenrechtliche Stellung der jüdischen Gemeinden in Deutschland. Tübingen: Mohr Siebeck. 
Denecke, Dietrich (2005): Soziale Strukturen im städtischen Raum: Entwicklung und Stand der sozialtopographischen Stadtgeschichtsforschung. In: Meinhardt, Matthias/Ranft, Andreas (Hrsg.): Die Sozialstruktur und Sozialtopographie vorindustrieller Städte. Beiträge eines Workshops am Institut für Geschichte der Martin-Luther-Universität Halle-Wittenberg am 27. und 28. Januar 2000. Berlin: de Gruyter, S. 123-137.

Dilcher, Gerhard (1996): Bürgerrecht und Stadtverfassung im europäischen Mittelalter. Köln: Böhlau.

Dirlmeier, Ulf/Fuhrmann, Bernd (2005): Räumliche Aspekte sozialer Ungleichheit in der spätmittelalterlichen Stadt. In: Vierteljahrschrift für Sozial- und Wirtschaftsgeschichte 92, H. 4, S. 424-439.

Eibach, Joachim u.a. (Hrsg.) (2015): Das Haus in der Geschichte Europas. Ein Handbuch. Berlin, Boston: de Gruyter.

Engelke, Thomas (1995): Regensburg und der Städtekrieg. In: Angerer, Martin/ Wanderwitz, Heinrich (Hrsg.): Regensburg im Mittelalter. Beiträge zur Stadtgeschichte vom frühen Mittelalter bis zum Beginn der Neuzeit. Regensburg: Univ.-Verl., S. 125-130.

Fleischmann, Peter (2008): Rat und Patriziat in Nürnberg. Die Herrschaft der Ratsgeschlechter vom 13. bis zum 18. Jahrhundert, Bd. 2: Ratsherren und Ratsgeschlechter Nürnberg: Verein für Geschichte der Stadt Nürnberg, S. 941-972.

Frenz, Barbara (2000): Gleichheitsdenken in deutschen Städten des 12. bis 15 . Jahrhunderts. Geistesgeschichte, Quellensprache, Gesellschaftsfunktion. Köln: Böhlau.

Gilomen, Hans-Jörg (1999): Spätmittelalterliche Siedlungssegregation und Ghettoisierung, insbesondere im Gebiet der heutigen Schweiz. In: Stadt- und Landmauern Abgrenzungen - Ausgrenzungen in der Stadt und um die Stadt. Unter Mitarbeit von Brigitt Sigel. Zürich: Vdf Hochschulverlag AG an der ETH Zürich, S. 85-106.

Gilomen, Hans-Jörg (2002): Städtische Sondergruppen im Bürgerrecht. In: Schwinges, Rainer Christoph (Hrsg.): Neubürger im späten Mittelalter. Migration und Austausch in der Städtelandschaft des alten Reiches (1250-1550). Berlin: Duncker und Humblot, S. 125-167.

Gilomen Hans-Jörg (2009): Juden in den spätmittelalterlichen Städten des Reichs. Normen -Fakten - Hypothesen, Trier: Kliomedia.

Greifenberg, Dominik (2015): Die Stadtmauer als Objekt korporativer Identifikation? Zur symbolischen und soziokulturellen Bedeutung der Stadtmauer für die Kölner Kommune im Hoch- und Spätmittelalter. In: Annalen des Historischen Vereins für den Niederrhein insbesondere das alte Erzbistum Köln, 218, S. 4594.

Groebner, Valentin (1993): Oekonomie ohne Haus. Zum Wirtschaften armer Leute in Nürnberg am Ende des 15. Jahrhunderts. Göttingen: Vandenhoeck und Ruprecht. 
Haverkamp, Alfred (1981): Die Judenverfolgungen zur Zeit des Schwarzen Todes im Gesellschaftsgefüge deutscher Städte. In: ders. (Hrsg.): Zur Geschichte der Juden im Deutschland des späten Mittelalters und der frühen Neuzeit. Stuttgart: Hiersemann, S. 27-93.

Hillebrand, Frank (2001): Differenz und Differenzierung in soziologischer Perspektive. In: Lutz, Helma/Wenning, Norbert (Hrsg.): Unterschiedliche verschieden. Differenz in der Erziehungswissenschaft. Opladen: Leske und Budrich, S. 47-70.

Holenstein, André (2008): Rituale der Vergewisserung: Der Eid als Mittel der Wahrheitsfindung und Erwartungsstabilisierung im Mittelalter und in der frühen Neuzeit. In: Bierende, Edgar/Oschema, Klaus/Bretfeld, Sven (Hrsg.): Riten, Gesten, Zeremonien: gesellschaftliche Symbolik in Mittelalter und Früher Neuzeit. Berlin: de Gruyter, S. 229-252.

Holtz, Eberhard (1993): Reichsstädte und Zentralgewalt unter König Wenzel (1376-1400). Warendorf: Fahlbusch.

Isenberg, Gabriele/Scholkmann, Barbara (Hrsg.) (1997): Die Befestigung der mittelalterlichen Stadt. Köln: Böhlau.

Isenmann, Eberhard (2014): Die deutsche Stadt im Mittelalter 1150 - 1550. Stadtgestalt, Recht, Verfassung, Stadtregiment, Kirche, Gesellschaft, Wirtschaft. 2., durchges. Aufl. Köln, Weimar, Wien: Böhlau.

Kießling, Rolf (2019): Im Spannungsfeld von lokalem Markt und europäischem Fernhandel - Oberschwaben als Wirtschaftsregion der Vormoderne. In: Hirbodian, Sigrid/ Kießling, Rolf/ Weber, Edwin Ernst (Hrsg.): Herrschaft, Markt und Umwelt. Wirtschaft in Oberschwaben 1300-1600. Stuttgart: Kohlhammer, S. 323-351.

Kohn, Karl (1978): Das hochmittelalterliche Judenviertel Nürnbergs. Eine topographische Rekonstruktion, Beilage zu Wolfgang Stromer: Die Metropole im Aufstand gegen König Karl IV. Nürnberg zwischen Wittelsbach und Luxemburg, Juni 1348 - September 1349. In: Mitteilungen des Vereins für Geschichte der Stadt Nürnberg 65, S. 55-90.

Koller, Heinrich (1989): Die mittelalterliche Stadtmauer als Grundlage städtischen Selbstbewusstseins. In: Kirchgässner, Bernhard/Scholz, Günther (Hrsg.): Stadt und Krieg. Sigmaringen: Thorbecke, S. 9-26.

Krause, Katharina (2019): The Legitimation of Council Rule Through Vedute of the City and Territory of Nürnberg from the Sixteenth to the Eighteenth Century: Visualizing Insecurity within an Image of Secured Order. In: Kreide, Regina/ Langenohl, Andreas (Hrsg.): Conceptualizing Power in Dynamics of Securitization. Beyond State and International System. Baden-Baden: Nomos, S. 175-238.

Kreutz, Bernhard (2005): Städtebünde und Städtenetz am Mittelrhein im 13. und 14. Jahrhundert. Trier: Kliomedia.

Marchal, Guy P. (2002): Pfahlburger, burgeois forains, buitenpoorters, bourgeois de roi. Aspekte einer zweideutigen Rechtsstellung. In: Schwinges, Rainer C. (Hrsg.): Neubürger im späten Mittelalter. Migration und Austausch in der Städtelandschaft des alten Reiches 1250-1550. Berlin: Duncker und Humblot, S. 333-367. 
Meier, Ulrich (1994): Regimen civitatis. Zum Spannungsverhältnis von Freiheit und Ordnung in alteuropäischen Stadtgesellschaften. In: Meier, Ulrich/Schreiner, Klaus (Hrsg.): Stadtregiment und Bürgerfreiheit. Handlungsspielräume in deutschen und italienischen Städten des Späten Mittelalters und der Frühen Neuzeit. Göttingen: Vandenhoeck und Ruprecht.

Moeller, Bernd (1971): Kleriker als Bürger. In: Festschrift für Hermann Heimpel. Bd. 2. Göttingen: Vandenhoeck und Ruprecht, S. 195-224.

Müller, Arnd (1968): Geschichte der Juden in Nürnberg: 1146 - 1945. Nürnberg: Stadtbibliothek Nürnberg.

Müller, Jörg R./Weber, Andreas (2016): Karl IV. und die Juden. In: Fajt, Jirí/ Hörsch, Markus (Hrsg.): Kaiser Karl IV. (1316-2016). Erste Bayerisch-Tschechische Landesausstellung. Prag: Národní galerie v Praze, S. 218-225.

Müller, Karlheinz (2004): Die Würzburger Judengemeinde im Mittelalter. Von den Anfängen um 1100 bis zum Tod Julius Echters (1617). Würzburg: Mainfränkischer Verein Kunst und Geschichte.

Müller, Karlheinz (Hrsg.) (2011): Die Grabsteine vom jüdischen Friedhof in Würzburg aus der Zeit vor dem Schwarzen Tod (1147 - 1346). Würzburg: Gesellschaft für Fränkische Geschichte.

Nicholson, Helen J. (2004): Medieval Warfare. Theory and Practice of War in Europe, 300-1500. Houndmills, Basingstoke, Hampshire, New York: Palgrave Macmillan.

Northemann, Yvonne (2011): Zwischen Vergessen und Erinnern. Die Nürnberger Klöster im medialen Geflecht. Petersberg: Imhof.

Oexle. Otto G./Hülsen-Esch, Andrea (Hrsg.) (1998): Die Repräsentation der Gruppen. Texte - Bilder - Objekte. Göttingen: Vandenhoeck und Ruprecht.

Piper, Ernst Reinhard (1982): Der Stadtplan als Grundriss der Gesellschaft. Topographie und Sozialstruktur in Augsburg und Florenz um 1500. Frankfurt: Campus Verlag.

Prietzel, Malte (2006): Kriegführung im Mittelalter. Handlungen, Erinnerungen, Bedeutungen [= Krieg in der Geschichte, 32]. Paderborn: Schöningh.

Rogger, Philippe/Schmid Keeling, Regula (Hrsg.) (2019): Miliz oder Söldner? Wehrpflicht und Solddienst in Stadt, Republik und Fürstenstaat 13.-18. Jahrhundert. Paderborn: Schöningh.

Rüther, Stefanie (Hrsg.) (2009): Integration und Konkurrenz. Symbolische Kommunikation in der spätmittelalterlichen Stadt. Münster: Rhema.

Rüther, Stefanie (2009a): Ungleiche Gegner? Zur Wahrnehmung und Bewertung kriegerischer Gewalt in den süddeutschen Städtekriegen. In: Stefanie Rüther (Hrsg.): Integration und Konkurrenz. Symbolische Kommunikation in der spätmittelalterlichen Stadt. Münster: Rhema, S. 37-60.

Rüther, Stefanie (2009b): Alltäglichkeit und Entgrenzung. Zum Bild des Krieges in der spätmittelalterlichen Chronistik. In: Emich, Birgit/Signori, Gabriela (Hrsg.): Kriegs/Bilder in Mittelalter und Früher Neuzeit. Berlin: Duncker und Humblot, S. 33-60. 
Rüther, Stefanie (2010), Production, perception et contrôle de l'espace dans la "Première ligue des villes souabes" (1376-1390). In: Histoire Urbaine 27, S. 143164.

Rüther, Stefanie (2014): Papierkriege? Schrift, Interaktion und Wehrpolitik im ausgehenden 14. Jahrhundert am Beispiel der Süddeutschen Städtekriege. In: Sawilla, Jan Marco/ Schlögl, Rudolf (Hrsg.): Medien der Macht und des Entscheidens. Schrift und Druck im politischen Raum der europäischen Vormoderne (14. 17. Jahrhundert). Hannover: Wehrhahn-Verlag, S. 33-50.

Rüther, Stefanie (2019): Der Bündnisfall. Ordnung und Organisation der Kriegführung des schwäbischen Städtebunds (1376-1390). In: Deigendesch, Roland/Jörg, Christian (Hrsg.): Städtebünde und städtische Außenpolitik. Träger, Instrumentarien und Konflikte während des hohen und späten Mittelalters. Ostfildern: Thorbecke, S. 213-232.

Rüther, Stefanie (2020): Ratsherren auf Kriegszug? Die Stellung der Hauptleute des Schwäbischen Städtebunds (1376-1390) zwischen Kompetenz und Kontrolle. In: Schmid, Regula/Hübner, Klara/Speich, Heinrich (Hrsg.): Bündnisdynamik. Träger, Ziele und Mittel politischer Bünde im Mittelalter. Berlin: Lit, S. 19-34.

Saur, Karl (1911): Die Wehrverfassung in schwäbischen Städten des Mittelalters. (Straßburg, Basel, Augsburg, Ulm, Rottweil, Überlingen, Villingen). Bühl: Konkordia A.G.

Scheutz, Martin (2016): Bürger und Bürgerrecht. Rechte und Pflichten und soziale Felder der mittelalterlichen und neuzeitlichen Bürger im Heiligen Römischen Reich. In: Gruber, Elisabeth u.a. (Hrsg.): Städte im lateinischen Westen und im griechischen Osten zwischen Spätantike und Früher Neuzeit. Topographie Recht - Religion. Wien, Köln, Weimar: Böhlau, S. 123-148.

Scheutz, Martin/Freitag, Werner (Hrsg.) (2021): Ein bürgerliches Pulverfass? Waffenbesitz und Waffenkontrolle in der alteuropäischen Stadt. Göttingen: Böhlau Verlag Köln.

Schmid, Regula (2019): Der Harnisch im Haushalt. Waffen als Indikatoren und als Triebkräfte sozialen Wandels in der mittelalterlichen Stadt. In: Heusinger, Sabine von/ Wittekind, Susanne (Hrsg.): Die materielle Kultur der Stadt in Spätmittelalter und Früher Neuzeit. Göttingen: Böhlau Verlag Köln, S. 205-224.

Schneider, Joachim (1999): „Denn wo das Ende böse ist“. Gründe und Begründungen für den ersten süddeutschen Städtekrieg in den Äußerungen der Chronisten. In: Brunner, Horst (Hrsg.): Der Krieg im Mittelalter und in der Frühen Neuzeit. Gründe, Begründungen, Bilder, Bräuche, Recht. Wiesbaden: Reichert, S. 139-182.

Schnelbögl, Fritz (1982, 1971): Topographische Entwicklung im 14. und 15. Jahrhundert. In: Pfeiffer, Gerhard (Hrsg.): Nürnberg, Geschichte einer europäischen Stadt. Unveränderter Nachdruck. München: Beck, S. 88-92.

Scholl, Christian (2012): Juden und Städtebünde. Annäherung an ein komplexes Beziehungsgeflecht. In: Hirbodian, Sigrid u.a. (Hrsg.): Pro multis beneficiis. Festschrift für Friedhelm Burgard. Forschungen zur Geschichte der Juden und des Trierer Raums. Trier: Kliomedia, S. 111-127 
Schubert, Alexander (2003): Der Stadt Nutz oder Notdurft? Die Reichsstadt Nürnberg und der Städtekrieg von 1388/89. Husum: Matthiesen.

Schubert, Alexander (2009): Zwischen Zunftkampf und Thronstreit. Nürnberg im Aufstand 1348/49. Bamberg: University of Bamberg Press.

Schultheiß, Werner (1969): Stadterweiterung und Vororte der Reichsstadt Nürnberg. In: Maschke, Erich/Sydow, Jürgen (Hrsg.): Stadterweiterung und Vorstadt. Protokoll über d. 6. Arbeitstagung d. Arbeitskreisesf. Südwestdt. Stadtgeschichtsforschung, Konstanz, 10. - 12. Nov. 1967. Stuttgart: Kohlhammer, S. 125-126.

Schultheiß, Werner (1972): Das Bürgerrecht der Königs- und Reichsstadt Nürnberg. Beiträge zur Verfassungsgeschichte der deutschen Städte. In: Festschrift für Hermann Heimpel zum 70. Geburtstag am 19. September 1971, Bd. 2. Göttingen: Vandenhoeck und Ruprecht, S. 159-194.

Simon-Muscheid Katharina (2000), Die Stadt als temporärer Zufluchtsort. Flüchtlinge und Flüchtlingspolitik im 15. Jahrhundert. In: Gilomen, Hans-Jörg/HeadKönig, Anne-Lise/ Radeff, Anne (Hrsg.): Migration in die Städte. Ausschluss, Assimilierung, Integration, Multikulturalität $[=$ Schweizerisches Jahrbuch für Wirtschafts- und Sozialgeschichte 16], S. 57-76.

Stromer, Wolfgang von (1970): Oberdeutsche Hochfinanz 1350-1450. 3 Bände (Vierteljahresschrift für Sozial- und Wirtschaftsgeschichte Beiheft 55). Wiesbaden: Steiner.

Tracy, James D. (Hrsg.) (2000): City Walls: The Urban Enceinte in Global Perspective. New York: Cambridge University Press.

Waever, Ole (1995): Securitization and Desecuritization. In: Lipschutz, Ronnie D. (Hrsg.): On Security. New York: Columbia University Press, S. 46-86.

Zeilinger, Gabriel (2007): Lebensformen im Krieg. Eine Alltags- und Erfahrungsgeschichte des süddeutschen Städtekriegs 1449/50. Stuttgart: Steiner. 